\title{
Structural changes after videogame practice related to a brain network associated with intelligence
}

\author{
Roberto Colom ， Ma Ángeles Quiroga , Ana Beatriz Solana , Miguel Burgaleta , \\ Francisco J. Román , Jesús Privado , Sergio Escorial , Kenia Martínez , Juan Álvarez-Linera , \\ Eva Alfayate , Felipe García , Claude Lepage , \\ Juan Antonio Hernández-Tamames ， Sherif Karama
}

\section{Introduction}

\subsection{Cognitive training and the brain}

Structural and functional changes in the brain induced by training are increasingly investigated in neuroscience (Boyke, Driemeyer, Gaser, Büchel, \& May, 2008; Draganski \& May, 2008; Ilg et al., 2008; Kelly \& Garavan, 2005; Klingberg, 2010; Lustig, Shah, Seidler, \& Reuter-Lorenz, 2009; Neubauer, Bergner, \& Schatz, 2010; Scholz, Klein, Behrens, \& Johansen-Berg, 2009). Training programs range from perceptual and motor activities
(Draganski et al., 2004; Houweling, Daffertshofer, van Dijk, \& Beek, 2008) to high-level working memory/executive updating mental processes (Dahlin, Stigsdotter Neely, Larsson, Bäckman, \& Nyberg, 2008; Hempel et al., 2004; Jolles, Grol, Van Buchem, Rombouts, \& Crone, 2010; Klingberg, 2010; Landau, Schumacher, Garavan, Druzgal, \& D'Esposito, 2004; Mohapel, Mundt-Petersen, Brundin, \& Frielingsdord, 2006; Olesen, Westerberg, \& Klingberg, 2004). Still further cognitive functions targeted by training are learning (Driemeyer, Boyke, Gaser, Büchel, \& May, 2008; Jausovec \& Jausovec, 2004; Kwok et al., 2011; Thomas et al., 2009) or analogical reasoning (Wartenburger, Heekeren, Preusse, Kramer, \& van der Meer, 2009).

After the seminal study by Maguire et al. (2000) showing larger posterior hippocampi in taxi drivers with respect to matched controls, several articles have been published reporting 
comparisons among groups with different talents and skills. Using voxel-based morphometry (VBM) Aydin et al. (2007) quantified greater cortical gray matter density in the left inferior frontal and bilateral inferior parietal cortices of mathematicians; these brain regions support mathematical reasoning and the observed relation increased with experience as a mathematician. From the analysis of diffusion tensor images (DTI) Bengtsson et al. (2005) reported increased white matter development after piano practicing in children, adolescents, and adults. Specifically, the pyramidal tract was more structured in pianists. Gaser and Schlaug (2003) compared musicians and controls with VBM analyses of the obtained images, finding significant gray matter volume differences in visual-spatial, auditory, and motor brain areas (left precentral gyrus, left Hesch's gyrus, and right superior parietal cortex). Schlaug, Norton, Overy, and Winner (2005) analyzed the presumed effect of music in the brain of developing children, using a longitudinal design. Findings were weak but focused in brain areas devoted to fine motor and melodic discriminations. Applying voxel-based analyses of DTI data, Lee et al. (2010) found greater fractional anisotropy (FA) indices in frontal, cingulum, and striato-thalamic areas in players trained in a complex game (Baduk). These brain structures are related to working memory, executive control, and problem solving. Using fMRI, Wan et al. (2011) identified specific activations of professional shogi's players in the parietal lobe (precuneus) during pattern recognition, and in the caudate nucleus for generating the next move on the board.

These two last studies connect previous research with the main focus of the present article, namely, the analysis of structural changes after videogame practice in brain networks probably related with intelligence. Haier, Karama, Leyba, and Jung's (2009) research is a recent and close antecedent. Using a pretest-posttest design, they analyzed cortical thickness changes and modifications in BOLD signals after gaining experience playing TETRIS. Twenty six females, that had no previous experience with this game, were considered in this study (15 were assigned to the practice group). Structural and functional images were obtained before and after practice ( $1.5 \mathrm{~h}$ per week on average during 3 months, $18 \mathrm{~h}$ total). The main findings revealed an increase in cortical thickness in frontal (BA 6) and temporal (BA 22/38) areas for the practice group. Further, BOLD signals decreased in frontal areas for the practice group, but none of the identified areas overlapped those detected in the structural analyses. The authors concluded that structural and functional changes might not be necessarily associated.

\subsection{The present study}

Here we also apply a pretest-posttest design. Twenty young females were carefully selected from a larger group of one hundred. General cognitive ability $(g)$ was assessed before recruiting and assigning participants to the practice and control groups. Matching them with respect to this psychological factor is important because of the cognitive nature of the game. Participants were scanned on week 1 . Afterwards, the practice group played the videogame four hours per week during four weeks (weeks 2-5). Instead of playing on-line (Haier et al., 2009; Owen et al., 2010) here participants practiced in the laboratory under strict supervision. Videogame performance data were gathered and analyzed after each session for control purposes - i.e. checking the expected improvements across sessions. On week 6 , participants were scanned again and they also completed a set of cognitive ability tests.

Professor Layton and The Pandora's Box (by Nintendo) was the chosen videogame because we have shown elsewhere significant requirements of general cognitive abilities (Quiroga et al., 2009,2011 ). The game is based on solving puzzles of increased mental complexity. These puzzles are interleaved with a story (Fig. 1). A total of 138 puzzles are included in the game and they demand abstract reasoning along with the mental manipulation of spatial, numerical, and verbal information.

Because of the demonstrated intelligence requirements, we predict changes in gray matter regions especially involved in high-level reasoning, but also in association areas given the need for combining verbal, numerical, and spatial content domains for getting a solution to the puzzle. Finally, white matter changes are also expected for tracts connecting relevant gray matter areas. Specifically, regions summarized by Colom et al. (2009), Colom, Karama, Jung, and Haier (2010), Gläscher et al. (2010), Jung and Haier's (2007) parieto-frontal theory of intelligence (P-FIT) are solid candidates: (a) the extrastriate cortex (BAs 18 and 19) and the fusiform gyrus (BA 37) involved in recognition, imagery, and elaboration of visual inputs - along with Wernicke's area (BA 22) - for analysis and elaboration of syntax of auditory information, (b) parietal BAs 39 (angular gyrus), 40 (supramarginal gyrus), and 7 (superior parietal lobule) for the integration and abstraction of sensory information, (c) frontal BAs 6, 9, 10, 45, 46, and 47 for problem solving, evaluation, and hypothesis testing. Nevertheless, there is a more restricted prediction based on Jung and Haier's (2007) underscoring of discrete regions of the dorsolateral prefrontal cortex (BAs 9, 45, 46, and 47) and the parietal cortex (BAs 7 and 40). Regarding white matter, the arcuate fasciculus is thought to play a relevant role for increasing the reliability of information transmission across these brain areas.

It is important to note that we use an active vs. passive control group design because we were not interested in comparing different videogames. If the interest is focused in comparing participants doing and not doing physical exercise, it makes little sense to compare jogging and body building. The difference between passive vs. active controls is crucial if the theory postulates advantages of aerobic over anaerobic exercise for preventing, say, cognitive decline. However, here we are not contrasting the effect of theoretically different videogames, but playing vs. not playing.

\section{Method}

\subsection{Participants}

As previously stated, twenty young females were selected from a larger sample of 100 individuals. ${ }^{1}$ They were psychology undergraduates and their mean age was $18.95(\mathrm{SD}=2.65)$. The following guidelines were used as including criteria: (a) no history of psychiatric or neurological illness, (b) little previous experience with videogames and none with Professor Layton and The Pandora's Box, ${ }^{2}$ (c) right handed, and (d) heterogeneity in their general cognitive ability. Written informed consent was

\footnotetext{
1 Participants were recruited at 'Colegio Universitario Cardenal Cisneros', Madrid, Spain.

${ }^{2}$ Note that it is much more easy to find females with no previous experience with videogames.
} 


\section{(A) Example of puzzle with spatial information processing requirements}

Puzzle 25: After years of bad business, a local zoo has finally run out of money to feed its animals. Bellies rumbling from days with no food, the animals make a plan to escape the zoo. After prying open the bars on their rusted cages, all the animals attempt to find their way through the mazelike walls of the zoo to the exit. Tap the picture of each animal you think made it safety out of the zoo, and then tap submit to answer. Just remember, an animal shows its true colors in the wild.
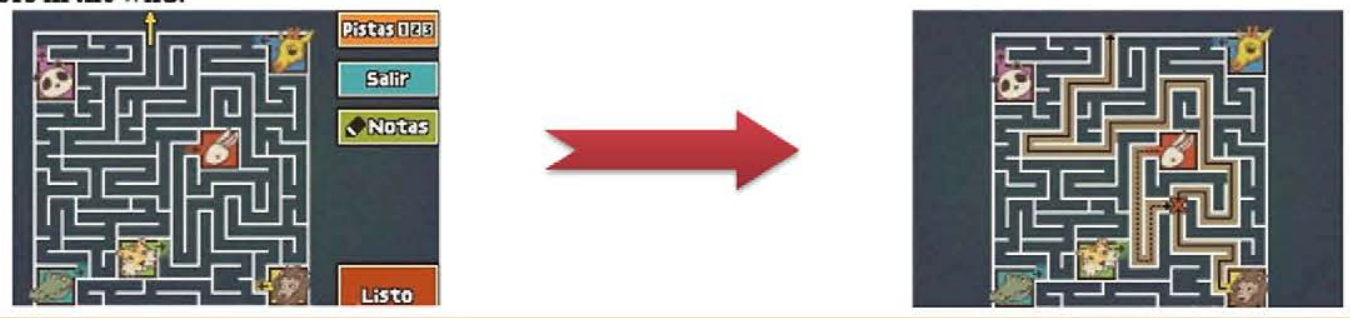

(B) Example of puzzle with numerical information processing requirements

Puzzle 38: You have four horses, all of which travel at different speeds. In traveling from point A to point B, these horses take one, two, four and six hours respectively. One day. you decide to move all your horses from point A to point B. However, you can only move a maximum of two horses at time, and you need to ride a horse back to point A each time you return to move your other horses. Knowing you can only move as fast as the lowest horse you're traveling with, what's the fewest number of hours it will take to complete your move?
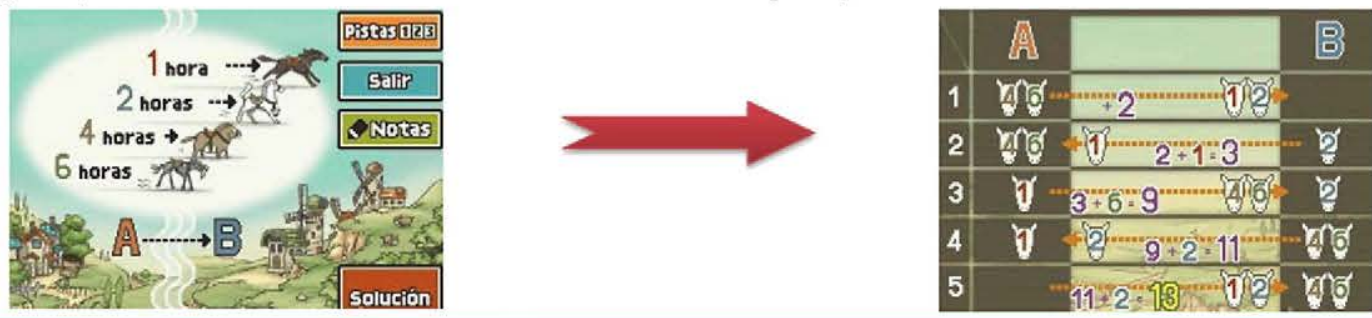

\section{(C) Example of puzzle with verbal information processing requirements}

Puzzle 24: After trying to fold a strip of paper in half, you notice that one side of the folder strip is one inch longer than the other. Determined to get it right, you fold the strip again, only to discover that now the other end of the folder strip is an inch longer than the other. Now that you've made two folds along your strip, figure out what the distance between the two folds is when expressed in tenths of an inch.
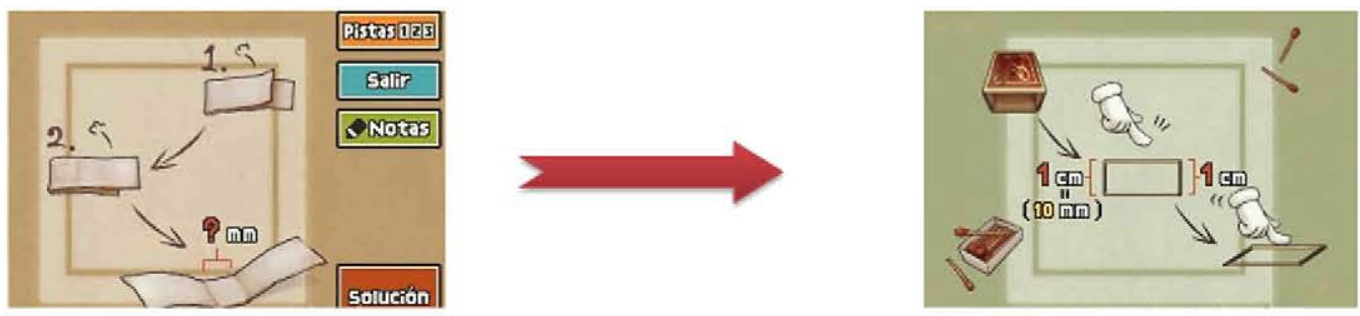

Fig. 1. Examples from 'Professor Layton and The Pandora's Box'. These examples are for spatial (A), numerical (B), and verbal (C) puzzles.

obtained in accordance with regulations of Fundación CIENFundación Reina Sofia (Madrid). The local ethical committee approved the study.

\subsection{Measures and procedure}

Firstly, the initial group of 100 participants completed a battery of four cognitive ability tests. These were screening versions of the Raven Advanced Progressive Matrices (RAPM)
Test (Raven, Raven, \& Court, 2004), along with the abstract reasoning (AR), spatial relations (SR), and verbal reasoning (VR) subtests of the Differential Aptitude Test (DAT) battery (Bennett, Seashore, \& Wesman, 2005). Only odd items were administered and accuracy scores were the dependent measure. Administration times were: $\mathrm{RAPM}=20 \mathrm{~min}$, DAT-AR $=10 \mathrm{~min}$, DAT-SR $=10 \mathrm{~min}$, and DAT-VR $=10 \mathrm{~min}$. The general ability score $(g)$ was computed summing the raw scores of the four individual tests. 
Secondly, brain images were acquired on a General Electric Signa 3 T MR Scanner (General Electric Healthcare, Farfield, CT) using a whole-body radiofrequency (RF) coil for signal excitation and quadrature 8-channel coil for reception. For the structural images analyzed here, a high-resolution 3D T1-weighted Gradient Echo-SPGR with parameters 1-mm slice thickness, $260 \times 260$ matrix, preparation time $=650 \mathrm{~ms}$, $\mathrm{TE}=4.2 \mathrm{~ms}$, TR $=9.2 \mathrm{~ms}$, flip angle $8^{\circ}$, complete volume with 154 sagittal slices. Diffusion-weighted images were also acquired for each participant. The pulse sequence was single-shot, diffusion-weighted, echo planar acquisition $(\mathrm{TR}=9000 \mathrm{~ms} ; \mathrm{TE}=$ minimum; $\mathrm{NEX}=2 ;$ matrix $=256 \mathrm{~mm} \times$ $256 \mathrm{~mm}$; FOV $=240 \mathrm{~mm} \times 240 \mathrm{~mm}$; slice thickness $=2.4 \mathrm{~mm}$; interslice gap $=0.3 \mathrm{~mm}$; in-plane resolution $=1 \mathrm{~mm}^{2} ; \quad b$ value $=1000 \mathrm{~s} / \mathrm{mm}^{2}$; diffusion gradient directions $=26$ ) .

Thirdly, the practice group completed the videogame. Participants played $4 \mathrm{~h}$ per week during a maximum of four weeks ( $16 \mathrm{~h}$ total). Practice time was distributed in two sessions every week. Playing was allowed in the laboratory only, because supervision by the experimenters was strictly required. At the end of each session, data were registered and analyzed for checking progress. Improvements were assessed according to puzzles of increasing complexity successfully solved. Participants had enough time for finishing the videogame at the end of the practicing period, but some of them ended without consuming the complete available period. Although they keep playing, puzzles solved were from a supplementary section of the game. The control group was explicitly instructed to follow their regular life, with an emphasis in avoiding playing any type of videogame. This was checked via interview before the second scanner.

Fourth, the whole group was scanned again within one week after the end of the practice period. The same scanning protocol was applied.

Finally, both the practice and control groups completed a new battery of cognitive ability tests comprised by screening versions of the same measures named above. However, even items were administered this time.

\subsection{VBM analyses}

These analyses were performed with SPM8 (Wellcome Trust Centre for Neuroimaging, 2009). Volumes for each participant were first manually aligned to the AC-PC for maximizing registration accuracy. These realigned images were bias corrected and segmented into gray matter, white matter, and CSF using default tissue probability maps (TPMs) provided with the software. Gray matter partitions were finally smoothed with a 12-mm FWHM isotropic Gaussian kernel to account for slight misalignments of homologous anatomical structures and to ensure statistical validity under parametric assumptions. Statistical analyses were based on a flexible factorial design ( 2 groups $\times 2$ conditions) including factors (subject, condition, group), subjects, and main effects and interactions (Fig. 2). From this design matrix positive (increments) and negative (decrements) gray matter changes were tested for the practice group with respect to the control group using a $p$ value of 0.005 uncorrected (Kwok et al., 2011). Total gray matter was a nuisance variable because of the interest in specific regional changes.

\subsection{Cortical surface and cortical thickness analyses}

Structural images were submitted to the CIVET pipeline for automated analyses (Ad-Dab'bagh et al., 2006). The specific stages for the analyses can be found in Karama et al. (2009, 2011) and involve (a) registration to MNI space, (b) generation of high-resolution hemispheric surfaces with 40,962 vertices each, (c) registration of surfaces to a high-resolution template, (d) application of a reverse of step 'a' allowing surface or thickness estimations in native space for each subject, (e) smoothing using a 20-milimeter kernel, and (f) computation of surface and thickness values at each vertex. Statistical analyses equivalent to those applied for VBM were computed using SurfStat (http:// www.math.mcgill.ca/keith/surfstat/) created for MATLAB 7 (The Math-Works, Inc.). Mean cortical surface area and mean cortical thickness were controlled.

\subsection{Tractography analyses}

These analyses were performed for estimating white matter indices in a set of projection, commissural, and association tracts (Fig. 3) for each participant and time-point. Tracts were selected based on their relevance to cognition, as reported in previous research (Chiang et al., 2011; Wakana, Jiang, NagaePoetscher, van Zijl, \& Mori, 2004; Yu et al., 2008). Firstly, all diffusion-weighted images were eddy current and motion corrected using FMRIB's Diffusion Toolbox (FDT, Smith et al., 2004; http://www.fmrib.ox.ac.uk/fsl/) prior to further processing. Non-brain tissue was extracted by applying a mask drawn manually over each subject's T2-weighted image. Secondly, a diffusion tensor model was fitted to DTI data for creating fractional anisotropy (FA) and first eigenvector $\left(v_{1}\right)$ maps, that were submitted to the FACT (Fiber Assignment by Continuous Tracking) algorithm, as implemented in the DTIStudio software (H. Jiang \& S. Mori, Johns Hopkins University, http://cmrm. med.jhmi.edu). This was done for the first scan only. Each tract's binary mask was generated in native space by two independent researchers via manual ROI placement, and interrater reliability (intra-class correlation) was above 0.95 for every tract. Thirdly, in order to avoid intra-rater reliability artifacts, tracts' binary masks generated in time 1 were translated to its corresponding native space in time 2 ; this was achieved by performing a six parameter registration of each subject's FA image in time 1 to that in time 2, by means of the FLIRT algorithm (Jenkinson, Bannister, Brady, \& Smith, 2002; Jenkinson \& Smith, 2001), and then applying the resulting transformation matrix to each tract's binary mask (nearest-neighbor interpolation). Results were visually inspected to ensure correct anatomical location of the registered tracts. For each tract, descriptive statistics were computed based on three common indices of white matter integrity: FA, axial diffusivity (AD) and radial diffusivity (RD). Finally, a paired $t$-test was performed and a false discovery rate (FDR) correction for multiple comparisons was applied.

\section{Results}

Table 1 shows descriptive statistics for the practice and control groups on the intelligence measures administered before scan 1 and after scan 2. Both groups do not show significantly different scores both at the pretest and posttest 


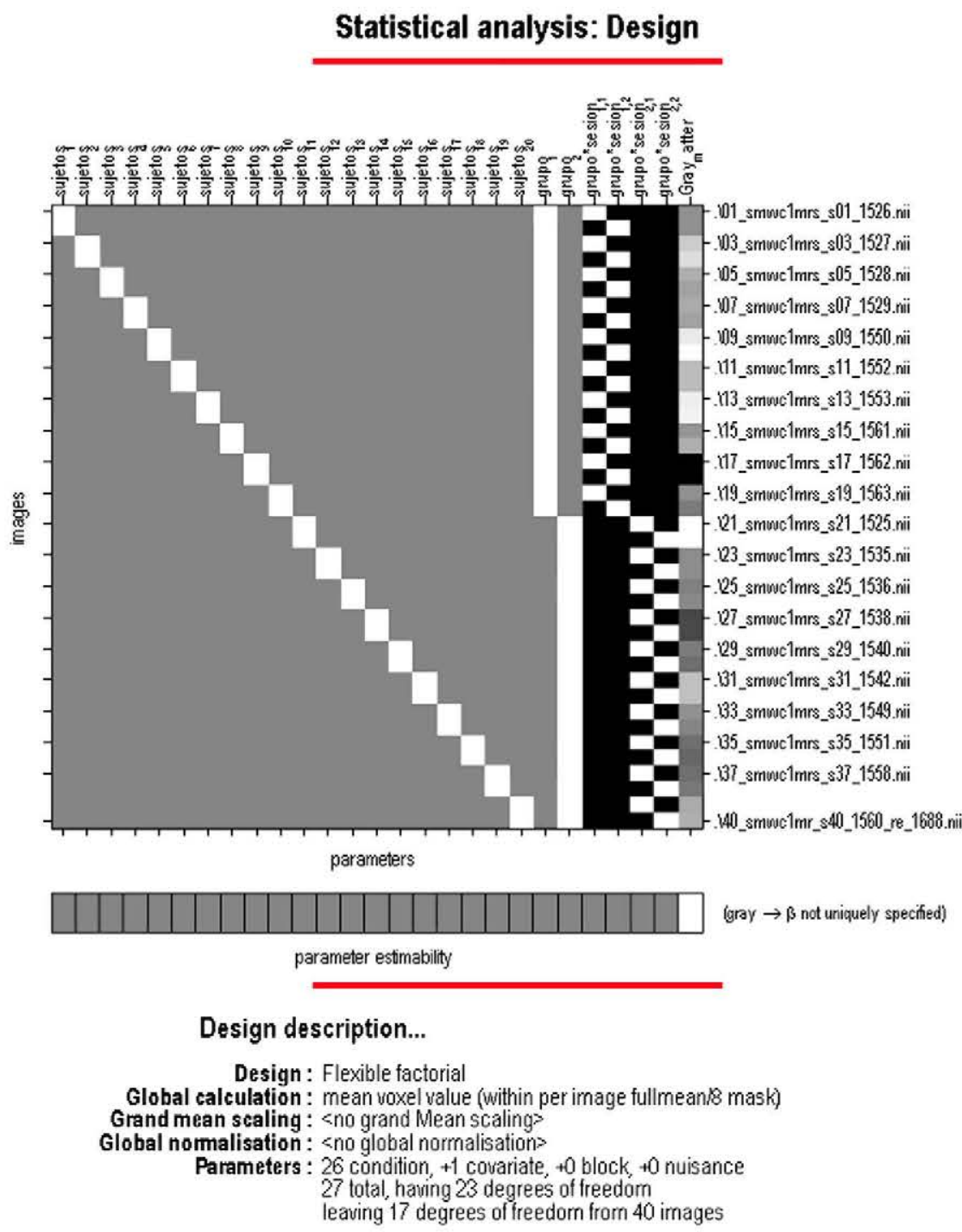

Fig. 2. Design matrix for VBM analyses. This matrix checks the double differences for the practice and control groups, as discussed in the Results section.

sessions [ $t(18)$ pretest $=-.336, p=.741, t(18)$ posttest $=$ $-.471, p=.643]$.

Fig. 4 depicts improvements of the practice group across sessions in 'Professor Layton and The Pandora's Box'. Participants improved from session to session, as required. Scores after hour 13 are not shown because several participants already finished the game.

Fig. 5 shows the 3D rendering for gray matter changes as revealed by the computed VBM analyses (Fig. 2). Significant positive changes (increments in regional gray matter from scan 1 to scan 2) are depicted in red, whereas negative changes (decrements in regional gray matter from scan 1 to scan 2) are shown in green.

Table 2 specifies brain regions (and corresponding approximate Brodmann area, BA) depicted in Fig. 5 along with the Talairach coordinates, cluster size, and $p$ values. $66 \%$ of the significant voxels belonged to the frontal lobes, $18 \%$ to the parietal lobes, $12 \%$ to the temporal lobes, and $4 \%$ to the occipital lobes. Both positive and negative changes involved frontal, parietal, and temporal regions, but occipital changes were negative only. Frontal regions comprise clusters in BAs 6 (premotor cortex), BA 9 (dorsolateral prefrontal cortex), and BA 10 (frontopolar region). Parietal regions were mainly concentrated in BAs $1 / 3$ (postcentral gyrus). Temporal clusters belong to BAs 22 (superior temporal gyrus) and BA 38 (temporopolar area). Occipital changes were concentrated in BA 18 (visual association cortex).

Cortical surface area and cortical thickness outputs are not shown because results were weak and not significant at $p<0.005$, uncorrected.

Finally, changes in white matter integrity were inspected. No significant changes in average FA $(p<0.01)$ for any of the explored tracts were detected, either in the practice or control group. Analysis of changes in robust maxima for FA also yielded no significant results. Analysis of axial and radial diffusivities again revealed no significant changes when average tract values were inspected, although robust maxima were found to significantly change in the practice group only. 


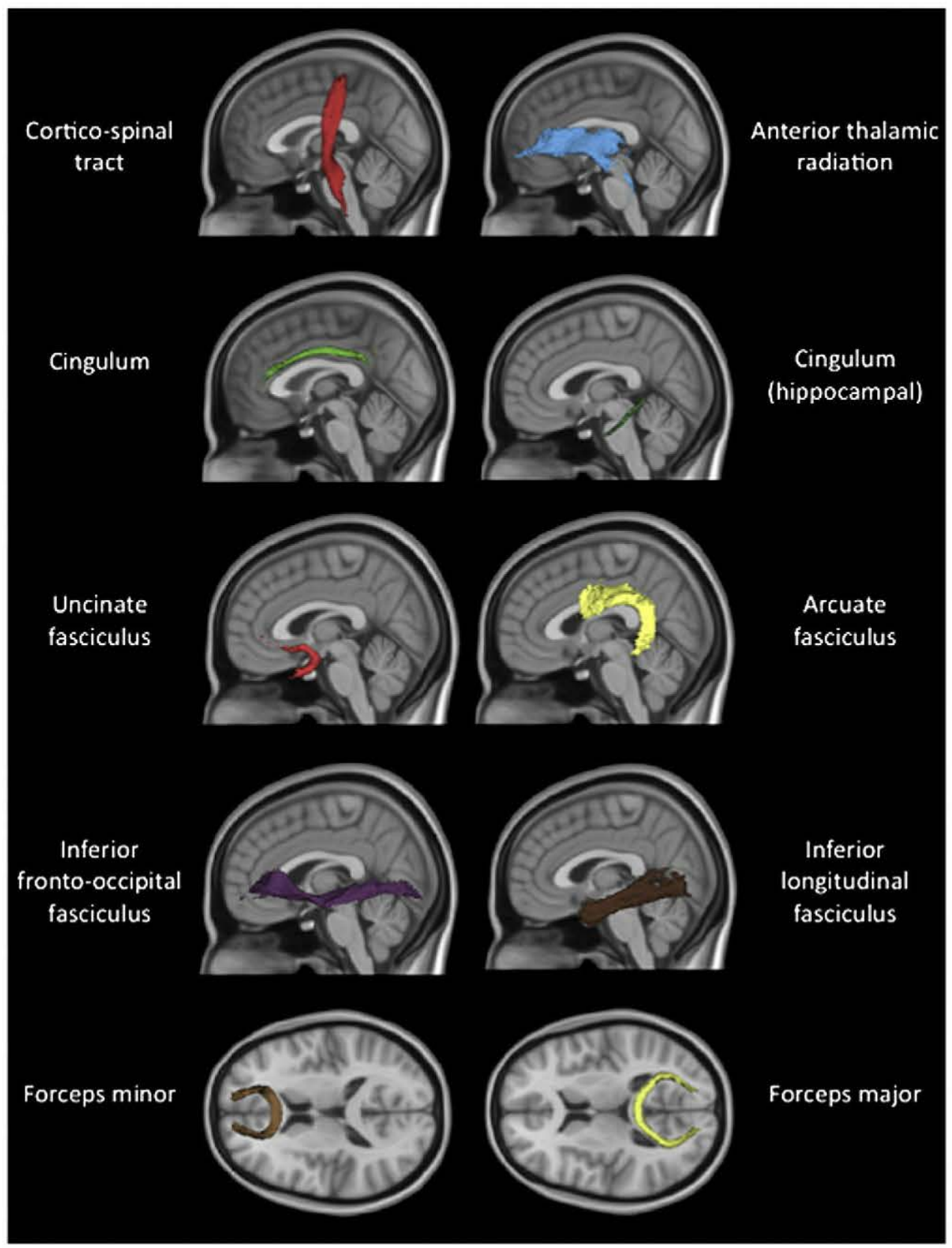

Fig. 3. White matter tracts analyzed for each participant and each scanner session. Note that these are the analyzed tracts. Obtained results are discussed in the Results section.

Table 1

Descriptive statistics for the practice $(\mathrm{N}=10)$ and control $(\mathrm{N}=10)$ groups at the pretest and posttest sessions. $\mathrm{SD}=$ standard deviation, $\mathrm{g}=$ general cognitive ability, RAPM = Raven Advanced Progressive Matrices Test, $\mathrm{AR}=$ abstract reasoning, $\mathrm{SR}=$ spatial relations, $\mathrm{VR}=$ verbal reasoning.

\begin{tabular}{|c|c|c|c|c|c|c|c|c|}
\hline & \multicolumn{4}{|c|}{ Pretest } & \multicolumn{4}{|c|}{ Posttest } \\
\hline & \multicolumn{2}{|c|}{ Practice } & \multicolumn{2}{|c|}{ Control } & \multicolumn{2}{|c|}{ Practice } & \multicolumn{2}{|c|}{ Control } \\
\hline & Mean & SD & Mean & SD & Mean & SD & Mean & SD \\
\hline$g$ & 46.1 & 9.9 & 47.7 & 11.3 & 48.7 & 13.3 & 50.3 & 11.0 \\
\hline RAPM & 10.6 & 1.6 & 10.6 & 2.4 & 12.2 & 2.6 & 12.3 & 2.3 \\
\hline AR & 12.0 & 4.2 & 13.9 & 4.5 & 11.2 & 4.1 & 11.2 & 3.9 \\
\hline SR & 12.7 & 4.1 & 11.9 & 4.9 & 13.6 & 4.9 & 14.5 & 4.9 \\
\hline VR & 10.8 & 3.4 & 11.3 & 2.5 & 11.7 & 3.5 & 12.3 & 2.2 \\
\hline
\end{tabular}

Specifically, an increase in the robust maximum axial diffusivity was observed in the right hippocampal cingulum ( $\mathrm{T}=3.548, \mathrm{p}=0.006$ ), whereas an increase in the robust maximum radial diffusivity was found in the left inferior longitudinal fasciculus ( $\mathrm{T}=3.891, \mathrm{p}=0.004$ ) (Fig. 6). Note that, after FDR correction for multiple comparisons, both effects remained significant at a trend level $(Q=0.108$ and 0.072 , respectively).

\section{Discusion}

Here a pretest-posttest design was applied for analyzing gray and white matter changes after a cognitive practice program 


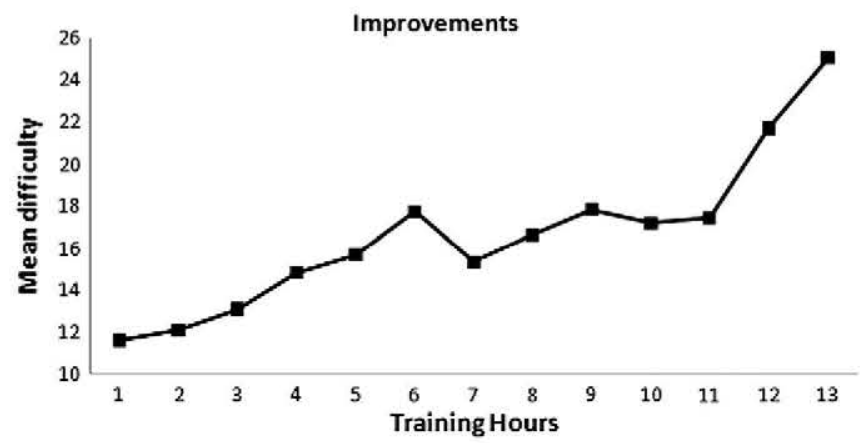

Fig. 4. Representation of improvements across practice sessions in 'Professor Layton and The Pandora's Box'. These improvements were computed from the complexity levels associated to the solved puzzles. Results are not shown after hour 13 because several participants already finished playing.

based on the commercial videogame Professor Layton and The Pandora's Box' (by Nintendo). This game raised varied cognitive challenges of increased complexity across sixteen sessions distributed in four weeks. Importantly, the practice group always played the game under strict supervision in the laboratory, instead of playing online (Haier et al., 2009; Owen et al., 2010).

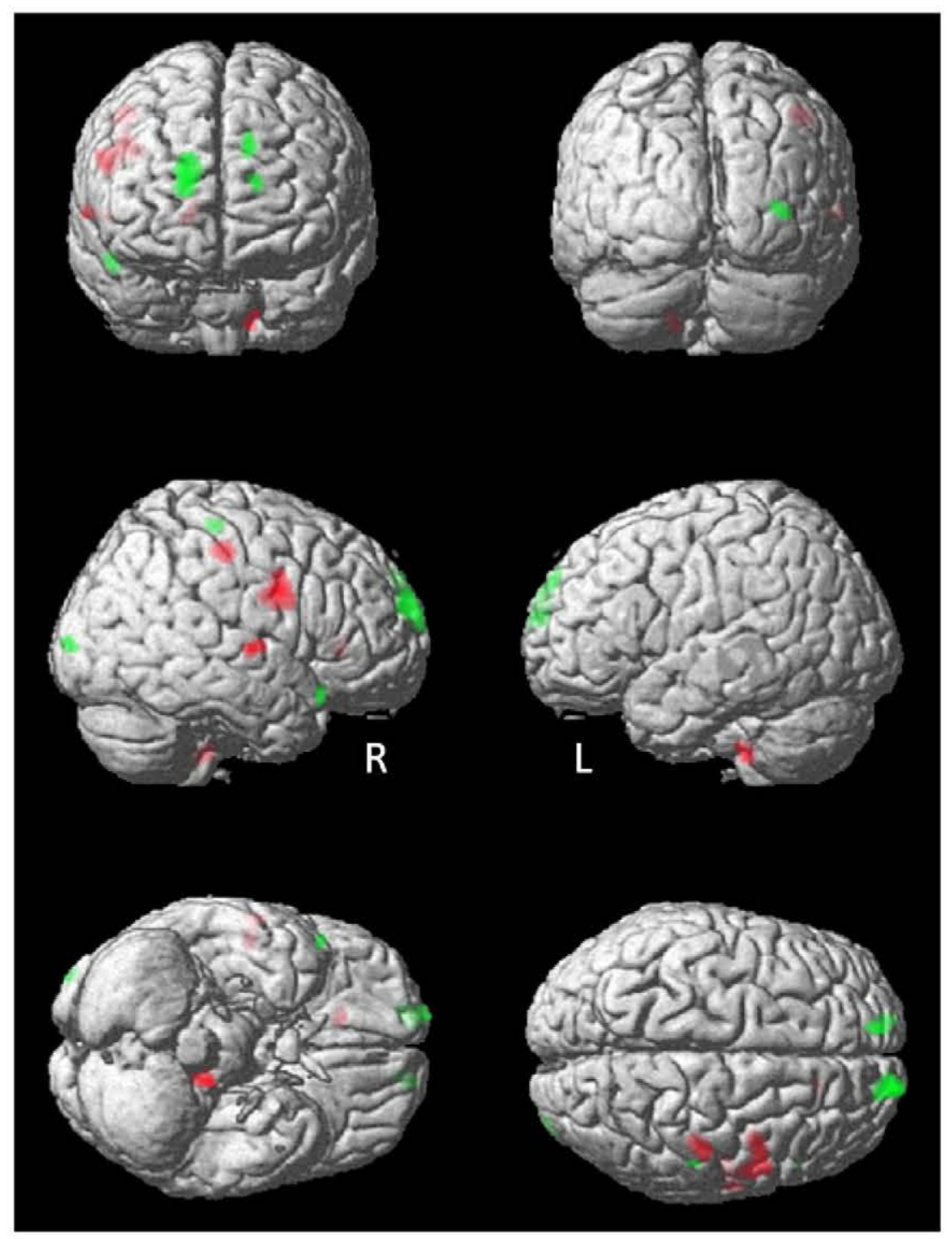

Fig. 5. Positive changes in gray matter (red) and negative changes (green) for the practice groups as compared with the control groups. $\mathrm{L}=$ left hemisphere, $\mathrm{R}=$ right hemisphere. 


\section{Table 2}

Gray matter changes for the practice group with respect to the control group ( red $=$ GM increments, green $=$ GM decrements $)$.

\begin{tabular}{|c|c|c|}
\hline Brain region & $x, y, z$ coordinates & Cluster size \\
\hline $\begin{array}{l}\text { Right inferior frontal } \\
\text { gyrus (BA 9) }\end{array}$ & 51525 & $\begin{array}{c}230 \\
p=0.001\end{array}$ \\
\hline $\begin{array}{l}\text { Right precentral gyrus } \\
\text { (BA 6) }\end{array}$ & 40129 & \\
\hline $\begin{array}{l}\text { Right postcentral gyrus } \\
\text { (BA 3) }\end{array}$ & $45-2145$ & $\begin{array}{c}82 \\
p=0.001\end{array}$ \\
\hline $\begin{array}{l}\text { Right superior temporal } \\
\text { gyrus (BA 22) }\end{array}$ & $51-122$ & $\begin{array}{c}69 \\
p=0.001\end{array}$ \\
\hline $\begin{array}{l}\text { Right superior temporal } \\
\text { gyrus (BA 22) }\end{array}$ & $61-84$ & \\
\hline $\begin{array}{l}\text { Right superior frontal } \\
\text { gyrus (BA 10) }\end{array}$ & 187011 & $\begin{array}{c}185 \\
p=0.002\end{array}$ \\
\hline $\begin{array}{l}\text { Right superior frontal } \\
\text { gyrus (BA 10) }\end{array}$ & 166119 & \\
\hline $\begin{array}{l}\text { Left superior frontal } \\
\text { gyrus (BA 9) }\end{array}$ & -125828 & $\begin{array}{l}45 \\
p=0.002\end{array}$ \\
\hline $\begin{array}{l}\text { Left superior frontal } \\
\text { gyrus (BA 10) }\end{array}$ & -146313 & $\begin{aligned} & 27 \\
p= & 0.003\end{aligned}$ \\
\hline $\begin{array}{l}\text { Right postcentral gyrus } \\
\text { (BA 1) }\end{array}$ & $55-2458$ & $p=0.001$ \\
\hline $\begin{array}{l}\text { Right superior temporal } \\
\text { gyrus (BA 38) }\end{array}$ & $5121-16$ & $\begin{aligned} & 20 \\
p= & 0.002\end{aligned}$ \\
\hline $\begin{array}{l}\text { Right middle occipital } \\
\text { gyrus (BA 18) }\end{array}$ & $36-958$ & $\begin{array}{c}30 \\
p=0.002\end{array}$ \\
\hline
\end{tabular}

Required performance improvements were detected (Fig. 4), and, afterwards, the practice group was compared with a nonplaying control group (matched by sex, age, handedness, and general cognitive ability) after the application of different image analysis protocols especially suited for computing the different brain tissues of interest. Specifically, gray matter was analyzed by optimized VBM and cortical surface area/cortical thickness indices, whereas white matter was considered after integrity values estimated from several projection, commissural, and projection tracts.

The most salient findings were derived from the VBM protocol. Fig. 5 depicts positive and negative changes for the practice group with respect to the control group. These changes were distributed across the brain, but they were mainly concentrated in frontal (66\%), parietal (18\%), and temporal ( $12 \%$ ) areas. Interestingly, Haier et al. (2009) reported thicker cortex in frontal BA 6 and temporal BAs 22/38. Here we also found gray matter changes in these frontal and temporal regions. Dorsolateral prefrontal changes (BA 9) were detected bilaterally, but gray matter increments were concentrated in the right side whereas decrements were localized in the left side. The superior frontal gyrus (BA 10), or frontopolar area, was also a relevant area. BAs 9 and 10 are involved in strategic processes for memory retrieval, attention, working memory, and executive function (Gilbert et al., 2006).

As noted above, and highlighted by Jung and Haier (2007) parieto-frontal regions are relevant for intelligence. Observed
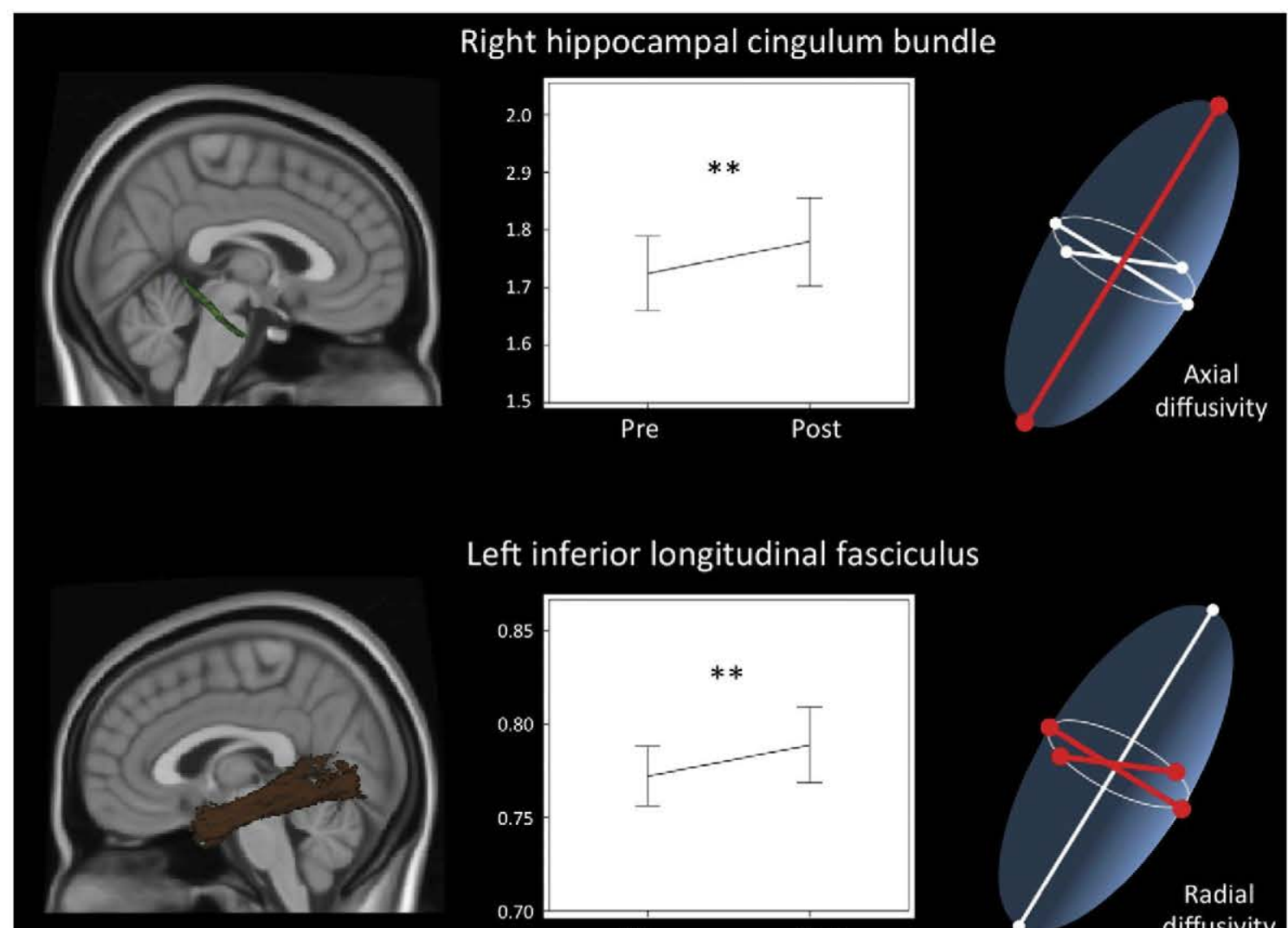

Left inferior longitudinal fasciculus

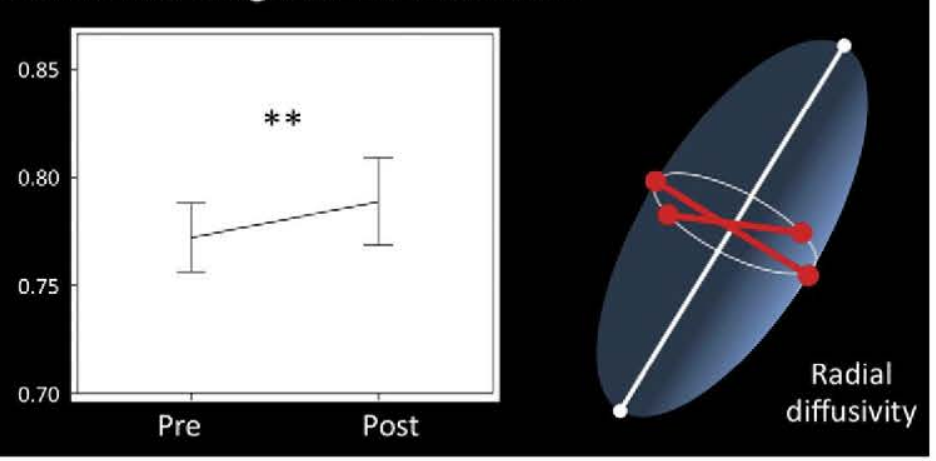

Fig. 6. Left panel shows white matter tracts where significant changes for the practice group were found. Middle panel depicts increments in axial and radial diffusivity from the pretest to the posttest. Right panel exemplifies these white matter indices. 
gray matter changes may reflect brain responsiveness to game requirements related to the integration of increasingly challenging verbal, numerical, and spatial requirements. The appreciated positive and negative changes might be also interpreted as dynamic adaptations of inter-related brain areas (Colom \& Thompson, 2011). Nevertheless, these brain changes are clearly not powerful enough for moving up intelligence tests' scores. As seen above (Table 1) there are no significant differences between the practice and control groups in measured intelligence at the posttest. Converting changes in the general intelligence index from the pretest to the posttest results in 3.3 and 3.4 IQ points for the practice and control groups respectively.

Nevertheless, it is important to underscore that intelligence was assessed here for control purposes. Control and practice groups are rarely matched for this psychological variable (Shipstead, Redick, \& Engle, 2012). Intelligence changes at the posttest were not predicted because Quiroga et al. (2009, 2011) have shown significant correlations between videogame performance and intelligence, but these researchers failed to find significant increments in intelligence tests' scores after gaming. The basic design applied here adds new evidence: solving problems of increased complexity comprised in a videogame does not impact on intelligence tests' scores even when brain changes related to gaming can be detected. This lack of significant changes can have different interpretations. Thus, for instance, brain changes were detected in some but not all the regions relevant for intelligence (Jung \& Haier, 2007). Further, perhaps sample size is an issue.

Biological signals do not always change in tandem with behavioral changes. The study by Neubauer et al. (2010) is an interesting example. In their research, cognitive training, based on real and virtual reality presentations, canceled behavioral sex differences in complex mental rotation tasks. However, these researchers failed to find a neurophysiological effect for this behavioral change in females. Increased neural efficiency (decreased activation) after practice was detected in males only.

On the other hand, cortical surface area/cortical thickness indices provided weak results, failing to replicate Haier et al. (2009) findings for TETRIS. These researchers also analyzed a sample of females of comparable size, but they found significant cortical thickness changes from the pretest to the posttest in the left superior frontal cortex and the left superior temporal lobe. Therefore, only the left side of the brain revealed significant gray matter changes, even when the nature of the videogame is highly spatial. It might be interesting to note, however, that these brain regions reported by Haier et al. (2009) for their videogame were also detected here for another videogame.

Results for white matter integrity revealed significant changes in the practice group only, although significance was at a trend level after FDR correction. Axial diffusivity (AD) increased after practice in the right hippocampal cingulum (HC), which includes fibers from the posterior cingulum that extend caudally to the parahippocampal gyrus (Makris \& Pandya, 2009; Makris et al., 2009). On the other hand, radial diffusivity (RD) increased in the left inferior longitudinal fasciculus (ILF), a white matter fiber bundle connecting occipital and temporal lobes (Mori, Wakana, Nagae-Poetscher, \& Van Zijl, 2005; Mori \& Zhang, 2006). AD accounts for the length of the main (longitudinal) axis of the diffusion ellipsoid, whereas RD captures diffusion variability that is perpendicular to the longitudinal axis. Importantly, these measures of diffusion have shown higher sensitivity than fractional anisotropy in previous studies on cognitive abilities (Burgmans et al., 2011; Tamnes et al., 2010). Nevertheless, increases in $A D$ are difficult to interpret, and it has been suggested that they might be related with alterations in axon diameter (Barazany, Basser, \& Assaf, 2009) and axonal coherence (Bennett, Madden, Vaidya, Howard, \& Howard, 2010). An increase in RD is usually interpreted in terms of myelin loss (Song et al., 2002; Sun, Liang, Schmidt, Cross, \& Song, 2007; Sun et al., 2006). Therefore, practice-related changes in the right $\mathrm{HC}$ might be explained by some kind of alteration in the spatial distribution of axons within the tract. Changes observed in the left ILF could be associated with a demyelination process that would encompass those left-hemisphere gray matter reductions reported above. Crucially, local reorganization of axons could also affect $\mathrm{AD}$ and $\mathrm{RD}$, and myelination and rewiring of crossing fibers are also potential explanations (Behrens, Berg, Jbabdi, Rushworth, \& Woolrich, 2007; Jbabdi, Behrens, \& Smith, 2010). Since integrity changes were not estimated here for crossing fibers, the results must be interpreted cautiously, and future research should implement DTI protocols able to track potential changes in secondary sets of fibers. Finally, it must be noted that significant changes were detected for robust maxima, and not by average values, thus suggesting a higher sensitivity for the former index. Therefore, further research should address what exact sub-regions of these tracts account for the observed changes.

In conclusion, we predicted gray and white matter changes highlighted by the parieto-frontal integration theory of intelligence (Jung \& Haier, 2007). Systematic changes were observed in the practice group as compared with the control group, which supports specific brain responsiveness to the practice program. Specifically, with respect to gray matter, volumetric changes, as revealed by the applied optimized VBM protocol, were mainly concentrated in the frontal lobes. Small white matter changes were also detected for the practice group only. These latter changes were hard to interpret. Finally, we acknowledge that VBM findings did not survive multiple comparisons correction and, therefore, there is a replication requirement. Nevertheless, we began with a very specific theoretical prediction and the reported results are generally consistent with it, thus reinforcing the main conclusion.

Ad-Dab'bagh, Y., Einarson, D., Lyttelton, O., Muehlboeck, J. -S., Mok, K., Ivanov, O., et al. (2006). The CIVET image-processing environment: A fully automated comprehensive pipeline for anatomical neuroimaging research. Neurolmage, 31. (pp. S-45): Supplement 1.

Aydin, K., Ucar, A., Oguz, K. K., Okur, O. O., Agayev, A., Unal, Z., et al. (2007). Increased gray matter density in the parietal cortex of mathematicians: A voxel-based morphometry study. American Journal of Neuroradiology, $28,1859-1864$.

Barazany, D., Basser, P. J., \& Assaf, Y. (2009). In vivo measurement of axon diameter distribution in the corpus callosum of rat brain. Brain, 132, 1210-1220.

Behrens, T. E., Berg, H. J., Jbabdi, S., Rushworth, M. F., \& Woolrich, M. W. (2007). Probabilistic diffusion tractography with multiple fibre orientations: What can we gain? Neurolmage, 34, 144-155.

Bengtsson, S. L., Nagy, Z., Skare, S., Forsman, L., Forssberg, H., \& Ullen, F. (2005). Extensive piano practicing has regionally specific effects on white matter development. Nature Neuroscience, 8(9), 1148-1150.

Bennett, I. J., Madden, D. J., Vaidya, C. J., Howard, J. H., Jr., \& Howard, D. V. (2010). Age-related differences in multiple measures of white matter integrity: A diffusion tensor imaging study of healthy aging. Human Brain Mapping, 31(3), 378-390. 
Bennett, G. K., Seashore, H. G., \& Wesman, A. G. (2005). Differential Aptitude Test (DAT-5). Madrid: TEA, S.A.

Boyke, J., Driemeyer, J., Gaser, C., Büchel, C., \& May, A. (2008). Training-induced brain structure changes in the elderly. Journal of Neuroscience, 28(28), 7031-7035.

Burgmans, S., Gronenschild, E. H. B. M., Fandakova, Y., Shing, Y. L., van Boxtel, M. P. J., Vuurman, E. F. P. M., et al. (2011). Age differences in speed of processing are partially mediated by differences in axonal integrity. Neurolmage, 55(3), 1287-1297.

Chiang, M., McMahon, K. L., Zubicaray, G. I., Martin, N. G., Hickie, I., Toga, A. W., et al. (2011). Genetics of white matter development: A DTI study of 705 twins and their siblings aged 12 to 29 . Neurolmage, 54, 2308-2317.

Colom, R., Haier, R. J., Head, K., Álvarez-Linera, J., Quiroga, M. A., Shih, P. C., et al. (2009). Gray matter correlates of fluid, crystallized, and spatial intelligence: Testing the P-FIT model. Intelligence, 37, 124-135.

Colom, R., Karama, S., Jung, R. E., \& Haier, R. J. (2010). Human intelligence and brain networks. Dialogues in Clinical Neuroscience, 12, 489-501.

Colom, R., \& Thompson, P. M. (2011). Understanding human intelligence by imaging the brain. In T. Chamorro-Premuzic, A. Furnham, \& S. von Stumm (Eds.), Handbook of individual differences. London: Wiley-Blackwell.

Dahlin, E., Stigsclotter Neely, A., Larsson, A., Bäckman, L., \& Nyberg, L. (2008), Transfer of learning after updating training mediated by the striatum. Science, 320, 1510-1512.

Draganski, B., Gaser, C., Busch, V., Schuierer, G., Bogdahn, U., \& May, A. (2004). Changes in grey matter induced by training. Nature, 427 , 311-312.

Draganski, B., \& May, A. (2008). Training-induced structural changes in the adult human brain. Behavioural Brain Research, 192, 137-142.

Driemeyer, J., Boyke, J., Gaser, C., Büchel, C., \& May, A. (2008). Changes in gray matter induced by learning - Revisited. PLoS One, 3(7), 2669-2669.

Gaser, C., \& Schlaug, G. (2003). Brain structures differ between musicians and non-musicians. Journal of Neuroscience, 23(27), 9240-9245.

Gilbert, S. J., Spengler, S., Simons, J. S., Steele, J. D., Lawrie, S. M., Frith, C. D., et al. (2006). Functional specialization within rostral prefrontal cortex (area 10): A meta-analysis. Journal of Cognitive Neuroscience, 18(6), 932-948.

Gläscher, J., Rudrauf, D., Colom, R., Paul, L. K., Tranel, D., Damasio, H., et al. (2010). The distributed neural system for general intelligence revealed by lesion mapping. PNAS, 107(10), 4705-4709.

Haier, R. J., Karama, S., Leyba, L., \& Jung, R. E. (2009). MRI assessment of cortical thickness and functional activity changes in adolescent girls following three months of practice on a visual-spatial task. BMC Research Notes, 2, 174, http://dx.doi.org/10.1186/1756-0500-2-174.

Hempel, A., Giesel, F. L., Garcia Caraballo, N. M., Amann, M., Meyer, H., Wüstenberg, T., et al. (2004). Plasticity of cortical activation related to working memory during training. The American Journal of Psychiatry, $161,745-747$

Houweling, S., Daffertshofer, A., van Dijk, B. W., \& Beek, P. J. (2008). Neural changes induced by learning a challenging perceptual-motor task Neurolmage, 41(4), 1395-1407.

Ilg, R., Wohlschläger, A. M., Gaser, C., Liebau, Y., Dauner, R., Wöller, A., et al. (2008). Gray matter increase induced by practice correlates with taskspecific activation: A combined functional and morphometric magnetic resonance imaging study. Journal of Neuroscience, 28(16), 4210-4215.

Jausovec, N., \& Jausovec, K. (2004). Differences in induced brain activity during the performance of learning and working-memory tasks related to intelligence. Brain and Cognition, 54, 65-74.

Jbabdi, S., Behrens, T. E., \& Smith, S. M. (2010). Crossing fibres in tract-based spatial statistics. Neurolmage, 49, 249-256.

Jenkinson, M., Bannister, P., Brady, J., \& Smith, S. (2002). Improved optimisation for the robust and accurate linear registration and motion correction of brain images. Neurolmage, 17(2), 825-841.

Jenkinson, M., \& Smith, S. (2001). A global optimization method for robust affine registration of brain images. Medical Image Analysis, 5, 143-156.

Jolles, D. D., Grol, M. J., Van Buchem, M. A., Rombouts, Serge A. R. B., \& Crone, E. A. (2010). Practice effects in the brain: Changes in cerebral activation after working memory practice depend on task demands. Neurolmage, $52,658-668$.

Jung, R. E., \& Haier, R. J. (2007). The parieto-frontal integration theory (P-FIT) of intelligence: Converging neuroimaging evidence. The Behavioral and Brain Sciences, 30, 135-187.

Karama, S., Ad-Dab'bagh, Y., Haier, R. J., Deary, I. J., Lyttelton, O. C., Lepage, C., et al. (2009). Positive association between cognitive ability and cortical thickness in a representative US sample of healthy 6 to 18 year-olds. Intelligence, 37, 145-155.

Karama, S., Colom, R., Johnson, W., Deary, I. J., Haier, R. J., Waber, D. P., et al. (2011). Cortical thickness correlates of cognitive performance accounted for by the general factor of intelligence in health children aged 6 to 18 . Neurolmage, 55, 1443-1453.
Kelly, A. M. C., \& Garavan, H. (2005). Human functional neuroimaging of brain changes associated with practice. Cerebral Cortex, 15, 1089-1102.

Klingberg, T. (2010). Training and plasticity of working memory. Trends in Cognitive Sciences, 14, 317-324.

Kwok, V., Niu, Z., Kay, P., Zhou, K., Mo, L., Jin, Z., et al. (2011). Learning new color names produces rapid increase in gray matter in the intact adult human cortex. www.pnas.org/cgi/doi/10.1073/pnas.1103217108.

Landau, S. M., Schumacher, E. H., Garavan, H., Druzgal, T., \& D'Esposito, M. (2004). A functional MRI study of the influence of practice on component processes of working memory. Neurolmage, 22, 211-221.

Lee, B., Park, J., Jung, W. H., Kim, H. S., Oh, J. S., Choi, C., et al. (2010). White matter neuroplastic changes in long-term trained players of the game of "Baduk" (GO): A voxel-based diffusion-tensor imaging study. Neurolmage, $52,9-19$.

Lustig, C., Shah, P., Seidler, R., \& Reuter-Lorenz, P. A. (2009). Aging, training, and the brain: A review and future directions. Neuropsychology Review, $19,504-522$.

Maguire, E. A., Gadian, D. G., Johnsrude, I. S., Good, C. D., Ashburner, J., Frackowiak, R. S. J., et al. (2000). Navigation-related structural change in the hippocampi of taxi drivers. PNAS, 97(8), 4398-4403.

Makris, N., \& Pandya, D. N. (2009). The extreme capsule in humans and rethinking of the language circuitry. Brain Structure \& Function, 213(3), 343-358.

Makris, N., Papadimitriou, G. M., Kaiser, J. R., Sorg, S., Kennedy, D. N., \& Pandya, D. N. (2009). Delineation of the middle longitudinal fascicle in humans: A quantitative, in vivo, DT-MRI study. Cerebral Cortex, 19(4), 777-785.

Mohapel, P., Mundt-Petersen, K., Brundin, P., \& Frielingsdord, H. (2006). Working memory training decreases hippocampal neurogenesis. Neuroscience, 142, 609-613.

Mori, S., Wakana, S., Nagae-Poetscher, L. M., \& Van Zijl, P. C. M. (2005). MRI atlas of human white matter. Amsterdam: Elsevier.

Mori, S., \& Zhang, J. (2006). Principles of diffusion tensor imaging and its applications to basic neuroscience research. Neuron, 51, 527-539.

Neubauer, A. C., Bergner, S., \& Schatz, M. (2010). Two vs, three dimensional presentation of mental rotation tasks: Sex differences and effects of training on performance and brain activation. Intelligence, 38, 529-539.

Olesen, P. J., Westerberg, H., \& Klingberg, T. (2004). Increased prefrontal and parietal activity after training of working memory. Nature Neuroscience, $7(1), 75-79$.

Owen, A. M., Hampshire, A., Grahn, J. A., Stenton, R., Dajani, S., Burns, A. S., et al. (2010). Putting brain training to the test. Nature, http://dx.doi.org/ 10.1038 /nature09042.

Quiroga, M. A., Herranz, M., Gómez-Abad, M., Kebir, M., Ruiz, J., \& Colom, R. (2009). Video-games: Do they require general intelligence? Computers in Education, 53, 414-418.

Quiroga, M. A., Román, F.J., Catalán, A., Rodríguez, H., Ruiz, J., Herranz, M., et al. (2011). Videogame performance (not always) requires intelligence. International Journal of Online Pedagogy and Course Design, 1(3), 18-32.

Raven, J., Raven, J. C., \& Court, J. H. (2004). Manual for Raven's Progressive Matrices and Vocabulary Scales. Pearson Assessment: San Antonio, TX.

Schlaug, G., Norton, A., Overy, K., \& Winner, E. (2005). Effects of music training on the child's brain and cognitive development. Annals of the New York Academy of Sciences, 1060, 219-230.

Scholz, J., Klein, M. C., Behrens, T. E. J., \& Johansen-Berg, H. (2009). Training induces changes in white-matter architecture. Nature Neuroscience, 12(11). 1370-1371.

Shipstead, Z., Redick, T. S., \& Engle, R. W. (2012). Is working memory training effective? Psychological Bulletin, http://dx.doi.org/10.1037/a0027473 (Advance online publication).

Smith, S. M., Jenkinson, M., Woolrich, M. W., Beckmann, C. F., Behrens, T. E. J., Johansen-Berg, H., et al. (2004). Advances in functional and structural MR image analysis and implementation as FSL. Neurolmage, 23(S1), 208-219.

Song, S. K., Sun, S. W., Ramsbottom, M. J., Chang, C., Russell, J., \& Cross, A. H. (2002). Dysmyelination revealed through MRI as increased radial (but unchanged axial) diffusion of water. Neurolmage, 17, 1429-1436.

Sun, S. W., Liang, H. F., Le, T. Q., Armstrong, R. C., Cross, A. H., \& Song, S. K. (2006). Differential sensitivity of in vivo and ex vivo diffusion tensor imaging to evolving optic nerve injury in mice with retinal ischemia. Neurolmage, 32, 1195-1204.

Sun, S. W., Liang, H. F., Schmidt, R. E., Cross, A. H., \& Song, S. K. (2007). Selective vulnerability of cerebral white matter in a murine model of multiple sclerosis detected using diffusion tensor imaging. Neurobiology of Disease, 28, 30-38.

Tamnes, C. K., Østby, Y., Walhovd, K. B., Westlye, L. T., Due-Tønnessen, P., \& Fjell, A. M. (2010). Intellectual abilities and white matter microstructure in development: A diffusion tensor imaging study. Human Brain Mapping, 31, 1609-1625.

Thomas, A. G., Marrett, S., Saad, Z. S., Ruff, D. A., Martin, A., \& Bandettini, P. A. (2009). Functional but not structural changes associated with learning: 
An exploration of longitudinal voxel-based morphometry (VBM). Neurolmage, 48, 117-125.

Wakana, S., Jiang, H., Nagae-Poetscher, L. M., van Zijl, P. C. M., \& Mori, S. (2004). Fiber tract-based atlas of human white matter anatomy. Radiology, $230,77-87$.

Wan, X., Nakatani, H., Ueno, K., Asamizuya, T., Cheng, K., \& Tanaka (2011). The neural basis of intuitive best next-move generation in board game experts. Science, 331, 341-346.
Wartenburger, I., Heekeren, H. R., Preusse, F., Kramer, J., \& van der Meer, E. (2009). Cerebral correlates of analogical processing and their modulation by training. Neurolmage, 48, 291-302.

Yu, C., Li, J., Liu, Y., Qin, W., Li, Y., Shu, N., et al. (2008). White matter tract integrity and intelligence in patients with mental retardation and healthy adults. NeuroImage, 40, 1533-1541. 